\title{
Supportive expressive group therapy did not prolong survival in metastatic breast cancer
}

\author{
Goodwin PJ, Leszcz M, Ennis M, et al. The effect of group psychosocial support on survival in metastatic breast cancer. \\ N Engl J Med 2001 Dec 13;345:1719-26.

\section{QUESTION: In women with metastatic breast cancer, does supportive expressive group therapy (SEGT) prolong survival, improve mood, and reduce pain?}

Sources of funding: Canadian Institutes of Health Research and Canadian Breast Cancer Research Initiative.

For correspondence: Dr P J Goodwin, Mount Sinai Hospital, Toronto, Ontario, Canada pgoodwin@mtsinai.on.ca

\section{Design}

Randomised \{allocation concealed\} $\}^{*}$, blinded (assessors of psychosocial outcomes), controlled trial with 12 months of follow up.

\section{Setting \\ 7 cancer centres in Canada.}

Patients

235 women (mean age 50 y) who had histological confirmation of breast cancer and had metastases outside of

\section{COMMENTARY}

In 1989, Spiegel et al reported a randomised trial indicating that women with metastatic breast cancer who received weekly SEGT for 1 year lived a mean of 18 months longer than those in the control group. ${ }^{1}$ A replication study was needed because of the possible relation between psychotherapy and prolongation of life, the clinical importance of the reported survival advantage, and controversies about methodological issues that might have influenced the findings. ${ }^{2}$ Although Spiegel claims that the oncology literature is evenly split on the psychotherapy-survival question, ${ }^{3}$ this is not the case for trials in metastatic breast cancer. Two randomised trials of psychosocial group therapy for women with metastatic breast cancer reported no group differences in survival. ${ }^{4}$ Goodwin et al's replication of Spiegel et al's study provides compelling evidence that SEGT does not prolong life.

Study strengths include the multicentre design with standardisation of Spiegel's intervention, ${ }^{1}$ comprehensive initial and ongoing manual based training for group leaders, thorough baseline patient assessments with frequent follow up, high participation rates, ample power to detect important group differences in survival, and statistical adjustment for important prognostic variables.

Although recruitment lasted 5 years, the survival data accumulated reduced the sample size requirement slightly. Significant interactions between group assignment and baseline psychological distress were found for the outcomes of mood and pain. Women who were more distressed at baseline benefited from the group intervention, whereas less distressed women did not. Unfortunately, insufficient follow up $(<80 \%)$ decreases the confidence that results for the secondary outcomes are representative of those who did not complete $\geqslant 1$ of the follow up questionnaires.

These are important findings for health professionals responsible for providing supportive care in the context of diagnosing and managing metastatic breast cancer. Patients and their families require information about the evidence on this subject. This may enhance patients' decision making about specific supportive care options most likely to meet their needs and accommodate their lifestyle choices. Future trials should evaluate the effectiveness of psychosocial therapies for metastatic breast cancer patients, especially those at high risk for emotional distress.

Bernice King, RN, MHSc, CRNH Clinical Nurse Specialist, Palliative Care Hamilton Health Sciences

Becky Fairfield, RN, CON(C) Supportive Care Nurse

Hamilton Regional Cancer Centre Hamilton, Ontario, Canada

1 Spiegel D, Bloom JR, Kraemer HC, et al. Effect of psychosocial treatment on survival of patients with metastatic breast cancer. Lancet 1989;2:888-91.

2 Fox B. Rejoinder to Spiegel $e$ t al. Psychooncology 1998;7:518-9.

3 Spiegel D. Mind matters - group therapy and survival in breast cancer. $N$ Engl J Med 2001;345:1767-8.

4 Cunningham AJ, Edmonds CV, Jenkins GP, et al. A randomised controlled trial of the effects of group psychological therapy on survival in women with metastatic breast cancer. Psychooncology 1998;7:508-17.

5 Edelman S, Lemon J, Bell D, et al. Effects of group CBT on the survival time of patients with metastatic breast cancer. Psychooncology 1999;8:474-81.

the breast and ipsilateral axilla. Exclusion criteria were central nervous system metastases; active psychosis, untreated major depression, or severe character disorder; planned participation in a therapist led support group for metastatic breast cancer outside of the study centre; residence $>1$ hour travel from the study centre; life expectancy $<3$ months; or inability to speak and read English. Follow up was complete for survival; follow up for psychosocial outcomes was $65 \%$ to $71 \%$.

\section{Intervention}

Women were allocated to SEGT $(n=158)$ or a control group $(n=77)$. SEGT consisted of weekly 90 minute meetings led by psychiatrists, psychologists, social workers, or nurse clinicians experienced in group therapy. Each group consisted of 8-12 women and 2 leaders. Women were supported and encouraged to speak about cancer and its effects on their lives (self image, roles, and relationships with family members, friends, coworkers, healthcare providers, and others), to interact with one another, and to support each other outside the group sessions. Women were asked to attend group sessions for $\geqslant 1$ year. Family and friends could attend a monthly 90 minute session. Control group women did not receive any psychological therapy. Educational material about breast cancer and its management, relaxation, and nutrition was provided to women in both groups every 4-6 months.

\section{Main outcome measures}

Survival. Secondary outcomes were changes in mood and pain.

\section{Main results}

Analysis was by intention to treat. Women who received SEGT did not differ from control group women for survival (median $17.9 v 17.6 \mathrm{mo}, \mathrm{p}=0.72$ ). The hazard ratio for death was 1.23 (95\% CI 0.88 to 1.72 ) after adjusting for presence or absence of progesterone or oestrogen receptors, time from first metastasis to randomisation, age at diagnosis, nodal stage at diagnosis, use or non-use of adjuvant chemotherapy, study centre, marital status, and baseline total mood disturbance score. The study had $85 \%$ power to identify a $15 \%$ difference between groups in survival. During the 12 months after randomisation, women in the SEGT group had greater improvement in $\operatorname{mood}(\mathrm{p} \leqslant 0.02)$ and less pain $(\mathrm{p}=0.04)$ than women in the control group, but these outcomes had $<80 \%$ follow up.

\section{Conclusion}

In women with metastatic breast cancer, supportive expressive group therapy did not prolong survival.

*Information provided by author. 